\title{
Photosynthetic Performance and Potency of Cannabis sativa L. Grown under LED and HPS Illumination
}

\author{
Michael W. Jenkins ${ }^{1,2}$, Curtis B. Livesay ${ }^{3}$ \\ ${ }^{1}$ Rubisco Research and Consulting, Sacramento, USA \\ ${ }^{2}$ Oaksterdam University, Oakland, USA \\ ${ }^{3}$ Dynamite Ag, Inc. \\ Email: mike@rubiscoconsulting.com
}

How to cite this paper: Jenkins, M.W. and Livesay, C.B. (2021) Photosynthetic Performance and Potency of Cannabis sativa L. Grown under LED and HPS Illumination. Agricultural Sciences, 12, 293-304. https://doi.org/10.4236/as.2021.123019

Received: February 24, 2021

Accepted: March 27, 2021

Published: March 30, 2021

Copyright $\odot 2021$ by author(s) and Scientific Research Publishing Inc. This work is licensed under the Creative Commons Attribution International License (CC BY 4.0).

http://creativecommons.org/licenses/by/4.0/

\begin{abstract}
Light-emitting diodes (LEDs) can be used as an energy efficient alternative to high-pressure sodium (HPS), which have historically been the standard for supplemental lighting in cannabis cultivation. However, there is a lack of scientific understanding in the cannabis industry regarding plant physiology, which has resulted in the adoption of cannabis cultivation methods based on hearsay rather than scientific research. The goals of this study were to 1) compare LED lighting options that are commonly used in the cannabis industry and 2) compare the top performing LED light with an industry standard HPS light. Specifically, three LED lights were compared (California Light Works ((SolarSystem 1100), BIOS Lighting (Icarus Gi2), and Fluence Bioengineering (now Fluence by Osram) (SPYDR xPLUS)), based on light distribution, leaf temperature, and photosynthetic performance indices. The LED versus HPS comparison was based on light response curves measured at photosynthetic photon flux densities (PPFD) of $(0,100,200,300,400,500$, $750,1000,1250,1500,1750$ and $\left.2000 \mu \mathrm{mol} \cdot \mathrm{m}^{-2} \cdot \mathrm{s}^{-1}\right)$, carbon assimilation rates (A) $\mu \mathrm{mol} \mathrm{CO} \mathrm{CO}_{2} \mathrm{~m}^{-2} \cdot \mathrm{s}^{-1}$ using a LiCor-6800 and resulting cannabinoid potency (THCA). The SPYDR xPLUS-Fluence by Osram had the highest performing LED light used in the LED comparison. At the suggested distance from bulb to canopy in the HPS versus LED comparison (6 inches for LEDs and $4 \mathrm{ft}$ for HPS), carbon assimilation rates displayed a $142 \%$ percent increase in plants grown under LED vs. HPS with average photon flux densities of 795 and 298 $\mu \mathrm{mol} \cdot \mathrm{m}^{-2} \cdot \mathrm{s}^{-1}$ for LED and HPS, respectively. All cultivars of Cannabis sativa L. showed increased cannabinoid potency when grown under LED illumination. The results of this study provide further insight regarding the selection of supplemental light to achieve maximum productivity of Cannabis sativa L.
\end{abstract}




\section{Keywords}

Cannabis, Light-Emitting Diode LED, High-Pressure Sodium HPS,

Carbon Assimilation, Light Response Curve, Cannabinoid, THC, Potency

\section{Introduction}

Light provides the energy required for photosynthesis and photosynthetic rate per unit leaf area is the driving force for all plant growth [1]. Light quality (color, wave-length), quantity (fluence rate), and photoperiod (duration of illumination) are dynamic and adjustable inputs that regulate plant growth and development (photomorphogenesis) to prevailing environmental conditions, which enables these light parameters to influence traits of horticultural interests, including plant stature, growth habits, the transition to flowering, and end point plant productivity [2] [3] [4]. Many horticultural crops are produced year-round, which requires the use of supplemental light. Among these crops is a burgeoning global cannabis market, which is projected to reach $\$ 103.9$ billion by 2024 [5]. Cannabis cultivators have many lighting options to choose from. Among those options is newer light technology as found in light-emitting diodes (LED) and the more traditional high-intensity discharge (HID) lights (i.e. high-pressure sodium (HPS) and/or metal halide). Given its formerly illicit nature and the associated stigma of cannabis cultivation the industry has suffered from a lack of scientific investigation. As such, cannabis cultivators have historically privileged anecdotal evidence and personal preference when it comes to answering questions such as the effectiveness of different lighting methods. In summary, the cannabis industry and $C$. sativa research have yet to provide the needed published peer reviewed results and data showing how $C$. sativa performs when grown using LED vs. HPS illumination.

Different environments will induce different phenotypic responses between and within specific genotypes or cultivars of cannabis, which can also exhibit variation in the production of secondary metabolites. When any number of environmental variables such as light, water, temperature, atmospheric conditions, or salinity in the root zone, are outside of optimal ranges, the immediate physiological response is the opening or closing of the stomata. This opening or closing of the stomata leads to a long list of physiological consequences including leaf temperature fluctuations and the extent of transpiration water loss, which have direct impacts on quality and quantity of secondary metabolite production and biomass productivity. Therefore, photosynthesis is a valuable physiological marker to aide in the determination of optimal environmental ranges for particular cannabis cultivars or the selection of cannabis cultivars for a particular environmental condition; and to further evaluate the response of cultivar(s) to a range of environmental stresses. It is well known that processes other than pho- 
tosynthesis can influence plant biomass production, but with respect to the impact of light, photosynthesis is often taken as the primary production determining process [6] [7].

HPS lamps emit adequate amounts of photosynthetically active radiation (PAR) and have been used as the primary lighting source for supplemental lighting in horticulture for decades; however, in addition to PAR, HPS lamps also emit long-wave (heat) radiation, which influences leaf temperature as well as the cultivation climate, which subsequently impacts plant processes such as transpiration and plant morphological development. Cultivating cannabis with LEDs for supplemental lighting is far more energy efficient than employing the traditional illumination methods such as HPS. Additionally, LEDs offer many other advantages, including high photoelectric conversion efficiencies, narrowband spectral light quality, adjustable light intensities, small mass and volume, long operating lifetimes, wavelength specificity, relatively cool emitting surfaces, and low thermal output. However, there is also a long list of potentially expensive and difficult obstacles to consider when designing a cannabis facility based on LED lighting or switching from HPS to LED. Among these concerns are relatively high initial capital cost, additional cultivation equipment needed (irrigation, tables, racks, etc.), learning curve for cultivators, modifications to air movement, heating, cooling and humidity budgets, and redeveloping growing standard operating procedures and modifying numerous cultivation practices.

How plants respond to the light under which plants are grown involves a complicated set of processes. Germination, seedling establishment, phenotypic expression, and the transition to reproduction are all regulated by light quantity, quality (spectral composition), direction, diurnal, and seasonal duration; all of which affect growth and development. Photoresponses to changes in spectral light composition and intensity evoke different morphogenetic and photosynthetic processes, which can be tailored to certain varieties of $C$. sativa and specific photoreceptors to optimize production and influence plant morphology and metabolism [8] [9] [10] and enable one to have more control over plant growth, development, and potency, which is of practical importance in the cannabis industry. Responses of certain plants to specific spectrums may sometimes be predictable based on published research; however, the overall plant response is generally difficult to predict due to the complicated interaction of many different responses [11]. In addition, many environmental stress conditions limit the ability of a plant to utilize light energy through photosynthesis so that excessive excitation of the photosystems can occur at moderate light intensities [12].

Photosynthetic carbon assimilation and secondary metabolite production in Cannabis sativa L. are temperature- and light-dependent processes [13]-[20]; however the elucidation of photosynthetic indices and cannabinoid concentration response to a variety of LED lighting sources and comparing LED to HPS light inputs, to the best of our knowledge, has yet to be explored. In this context, the overall hypothesis of this study was that $C$. sativa grown under an LED light 
source that provides more optimal photosynthetic photon flux density (PPFD) and reduced thermal heat load, will photosynthetically outperform and have increased cannabinoid potency compared to $C$. sativa grown under a traditional HPS light. Specific objectives included: 1) to quantify the uniformity of light emitted and cannabis' ability to harness and photosynthetically use the light provided throughout the canopy area by different LED options; 2) investigate a range of cannabis cultivars with differing morphological characteristics to determine if specific cultivars perform differently when grown under LED versus HPS lights; and 3) test the photosynthetic performance/productivity and cannabinoid potency between cannabis grown using LED versus HPS lights. The results of this study provide further insight regarding the selection of supplemental light to achieve maximum productivity of Cannabis sativa L.

\section{Materials and Methods}

\subsection{Plant Material and Growth Conditions}

Plants of C. sativa L. including cultivars (Northern Lights, Money Pug, Sour Diesel, Larry OG, Dead Head, Golden Goat, Witches Weed, BioChem, Sage and Sour, Tangerine Haze, and Space Queen) were grown in Denver Colorado, USA, at a state approved cannabis cultivation facility. The morphological groupings used in this study are representative of general characteristics used when genetics/cultivars are chosen for commercial scale cannabis production. Experiments were carried out to assess light distribution, photosynthetic performance, and potency of $C$. sativa in response to HPS and LED illumination.

All plant cultivars were originally obtained and started from seeds and grown under metal halide lights at $250 \mu \mathrm{mol} \cdot \mathrm{m}^{-2} \cdot \mathrm{s}^{-1}$ under similar conditions until sex was established and single "mother" plants were isolated and all clones were established form the same mother plants. Clones were taken and kept in conditions suitable for root initiation (high humidity, hormones, rockwool, heating pads, T12 fluorescent illumination) for 14 days until roots were clearly established. Plants were then transplanted into 1-gallon pots and kept in vegetative light cycle (18 hours of light and 6 hours of darkness) using metal halide illumination for 4 weeks and then transplanted to 5-gallon pots for the duration of their life cycle with the same grow medium. They were watered daily with a complete nutrient solution and kept in a grow room at $20^{\circ} \mathrm{C}$ to $30^{\circ} \mathrm{C}$ and $50 \%$ to $80 \%$ RH. Plants were grown in 60\% Plagron coco coir and 40\% Persolite \#6 perlite during their life cycle after the cloning period. Abiotic conditions were adjusted weekly and biotic treatments were performed periodically to maintain maximum productivity.

\subsection{Comparing LED Lights}

California Light Works (SolarSystem 1100), BIOS Lighting (Icarus Gi2), and Fluence Bioengineering (now Fluence by Osram) (SPYDR xPLUS) were chosen 
for LED comparison. Each of the LED models was designed to cover a $4 \mathrm{ft} \times 4 \mathrm{ft}$ (16 square foot) canopy area with uniform light and high PPFD.

\subsection{Light Distribution}

Carbon assimilation rate $(A) \mu \mathrm{mol} \mathrm{CO}_{2} \mathrm{~m}^{-2} \cdot \mathrm{s}^{-1}$ and Leaf Qabs $\mu \mathrm{mol} \cdot \mathrm{m}^{-2} \cdot \mathrm{s}^{-1}$ measurements (Leaf Qabs was chosen for these initial measurements since it provides an estimate of PPFD absorbed by the leaf) were collected using a LiCor 6800 (as identified in the photosynthetic measurements section) at designated locations (middle, corner, side) of the 16 square foot canopy area, to determine light distribution, uniformity, and photosynthetic response to light provided by three different LED sources.

\subsection{LED versus HPS}

Individual plants were randomly assigned into two light treatments using different types of illumination during flowering stage (12 hours light and 12 hours dark) ePapillion $1000 \mathrm{w}$ Double Ended HPS (PPFD, radiation focused between $550-620 \mathrm{~nm}$ ) and Fluence Bioengineering (now Fluence by Osram) SPYDR xPLUS LED (PPFD, radiation focused between $400-680 \mathrm{~nm}$ ) lights.

\subsection{Photosynthetic Measurements}

The response of net photosynthesis $(A)$ to incident PPFD was measured on third order sets of leaves of five randomly selected plants from each cultivar per treatment. Leaf level gas-exchange measurements were conducted using a portable open-mode photosynthesis system (model LI-6800, Li-Cor, Lincoln, NE, USA) with a $2 \times 3 \mathrm{~cm}$ light source chamber (6400-02B LED, Li-Cor Inc.) and an external $\mathrm{CO}_{2}$ source (set to $900 \mu \mathrm{mol} \cdot \mathrm{mol}^{-1}$ for all photosynthetic measurements) for the light response curves. Measurements were made with the leaf cuvette temperature controlled to within $\pm 1^{\circ} \mathrm{C}$ of the target temperature. An airflow rate of $300 \mathrm{mmol} \cdot \mathrm{s}^{-1}$ was used to maintain a significant differential of $\mathrm{CO}_{2}$ and $\mathrm{H}_{2} \mathrm{O}$ concentration between the reference and sample infrared gas analyzers. For each measurement, fully mature, light saturated, healthy leaves were clamped into the chamber resulting in the entire $2 \times 3 \mathrm{~cm}$ chamber area full of leaf material and the chamber gaskets were coated with G35 Qubitac Sealant (Qubit Systems Inc., Kingston, ON, Canada) to minimize $\mathrm{CO}_{2}$ leakage. During all measurements, vapor pressure deficit (VPD) was allowed to vary with conditions inside the cuvette. Measurements were recorded after stability criteria were met for $3-5 \mathrm{~min}$.

\subsection{Light Response Curves}

Light response curves measured using the ePapillion 1000 w Double Ended HPS and Fluence Bioengineering (now Fluence by Osram) SPYDR xPLUS LED lights were conducted using the clear LiCor chamber without the internal light source 
attachment and adjusting the distance from bulb to measured leaf until the needed PPFD was achieved for each light level (0, 100, 200, 300, 400, 500, 750, $1000,1250,1500,1750$ and 2000$) \mu \mathrm{mol} \cdot \mathrm{m}^{-2} \cdot \mathrm{s}^{-1}$. The order of individuals measured for each response curve was random. Light response curves were conducted on a subset of 7 cultivars.

\subsection{Cannabinoid Concentration (THCA)}

PhytaTech performed liquid extraction and analyses, in accordance with PhytaTech SOP-020, to monitor the product chemotype. Analysis on PhytaTech's potency assay, performed on Agilent 1100 HPLCs, is validated annually according to WHO and AOAC validation criteria. Quantitation was performed using reverse phase HPLC columns, DAD detection at $284 \mathrm{~nm}$ and $220 \mathrm{~nm}$, quaternary gradient pumps, column ovens and automatic autosamplers. Mobile phases include acidified (formic acid) methanol and acidified water, both LC grade. All analysis was performed with chemstation software and the laboratory's custom-built LIMS system.

\subsection{Data Analysis}

The data obtained were statistically analyzed using GraphPad Prism version 6.0d for Mac OS X (GraphPad Software, San Diego California USA) the results are presented as mean \pm standard error. Comparisons of photosynthetic parameters and secondary metabolites between LED and HPS were made using a $T$-test.

\section{Results}

The first part of this study we compared three leading LED manufactures/models including Fluence (SPYDR xPLUS), BIOS (Icarus Gi2), and California Light Works (SolarSystem 1100). Fluence SPYDR xPLUS light provided the most uniform PPFD absorbed by leaf of the three models tested (Figure 1). More specifically, the SPYDR xPLUS had the most similar Leaf Qabs measured at the middle, corner, and side of the $4 \mathrm{ft} \times 4 \mathrm{ft}$ canopy area (between 300 and 600 Leaf Qabs $\mu \mathrm{mol} \cdot \mathrm{m}^{-2} \cdot \mathrm{s}^{-1}$ ), which yielded $A$ between 20 and $30 \mu \mathrm{mol} \mathrm{CO} \mathrm{m}^{-2} \cdot \mathrm{s}^{-1}$ (Figure 1 ). The side and corner light measurements for BIOS and California Light Works were below 200 Leaf Qabs $\mu \mathrm{mol} \cdot \mathrm{m}^{-2} \cdot \mathrm{s}^{-1}$ with $A$ rates below $15 \mu \mathrm{mol} \mathrm{CO} \mathrm{m}^{-2} \cdot \mathrm{s}^{-1}$ (Figure 1). California Light Works had the least uniform distribution of light (Figure 1). Plants grown under Fluence SPYDR xPLUS lights offered the most consistency in terms of leaf temperature (Figure 2). BIOS and California Light Works lights caused leaf temperatures to rise to values approaching and exceeding $30^{\circ} \mathrm{C}$ with leaves measured at varying locations (Figure 2). Given the superiority of consistency of the Fluence SPYDR xPLUS relative to the other LED lights tested, the SPYDR xPLUS was utilized for comparison to HPS lighting. None of these physiological parameters were significantly different between any of the cultivars used in this study; therefore, data was pooled (data not shown). 


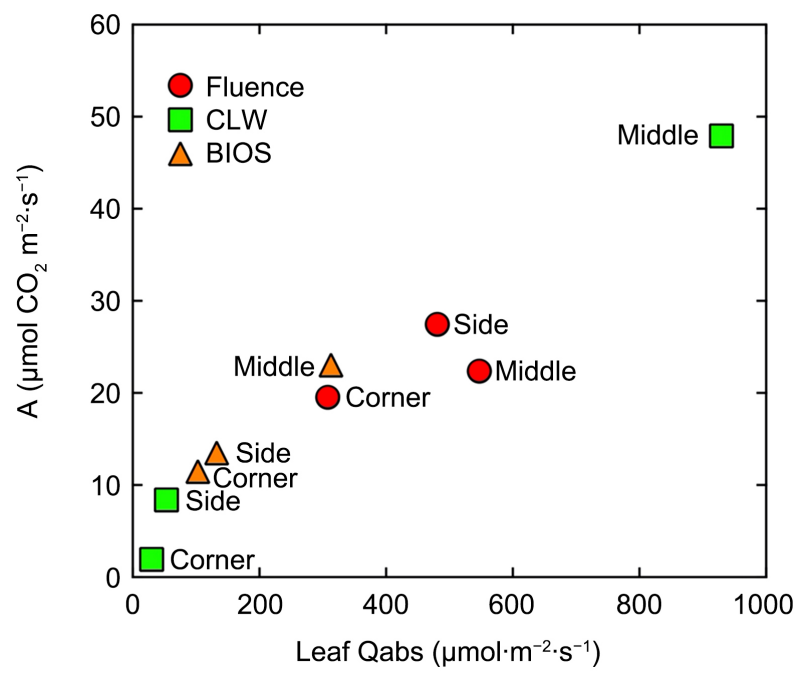

Figure 1. Variations in carbon assimilation rate $(A)$ in $C$. sativa with varying photosynthetic photon flux densities absorbed by leaf (Leaf Qabs) provided by three different LED sources Fluence (Fluence Bioengineering (now Fluence by Osram) (SPYDR xPLUS), CLW (California Light Works (SolarSystem 1100), BIOS (Icarus Gi2). Canopy space covered by each light was $4 \mathrm{ft} \times 4 \mathrm{ft}$ (16 sqft) area. Labels next to symbols represent location within the canopy where measurements were conducted (Middle, Corner, Side).

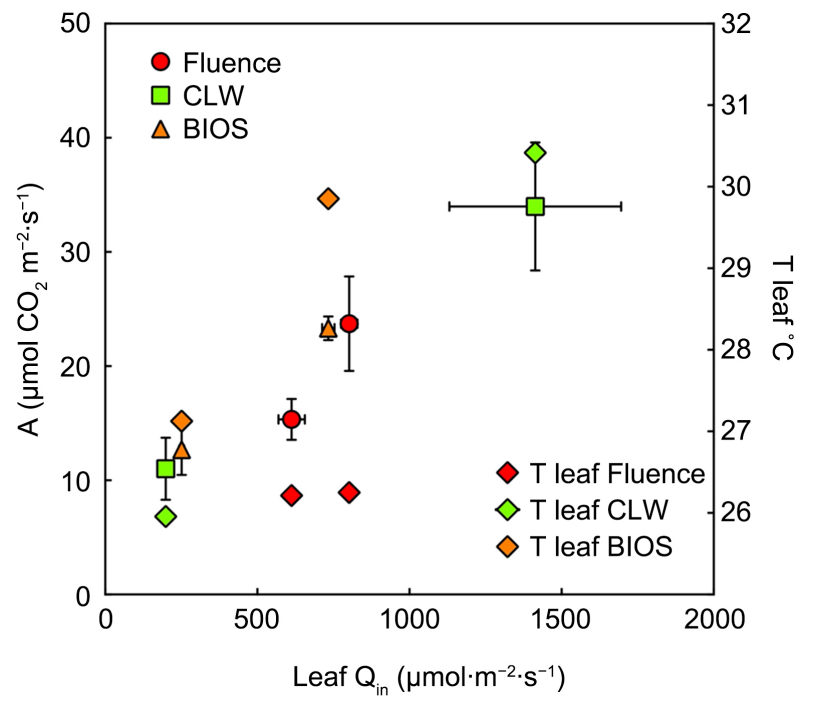

Figure 2. Variations in carbon assimilation rate $(A)$ and leaf temperature (T leaf) with varying photon flux density (Leaf $Q_{\text {in }}$ ) provided by different LED light sources Fluence (Fluence Bioengineering (now Fluence by Osram) (SPYDR xPLUS), CLW (California Light Works (SolarSystem 1100), BIOS (Icarus Gi2) for C. sativa.

The second part of this study was to compare the top performing LED light source (Fluence SPYDR xPLUS) and a leading industry standard HPS light source (ePapillion). Light response curves measured on plants grown under LED and HPS lights failed to light-saturate; however, photosynthetic rates did begin to level off once Leaf $Q_{\text {in }}$ exceeded $1000 \mu \mathrm{mol} \cdot \mathrm{m}^{-2} \cdot \mathrm{s}^{-1}$ (PPFD) (Figure 3). At the distance these light companies suggest from bulb to canopy (6 inches LED and 4 
ft HPS) HPS averaged $298 \mu \mathrm{mol}$ PPFD and $11.26 A \mu \mathrm{mol} \mathrm{CO} \mathrm{m}^{-2} \cdot \mathrm{s}^{-1}$, LED averaged $795 \mu \mathrm{mol}$ PPFD and $27.26 A \mu \mathrm{mol} \mathrm{CO} \mathrm{m}^{-2} \cdot \mathrm{s}^{-1}$ (Figure 3). THCA\% were significantly different between flower grown using HPS lights (19.67\% THCA mean) and LED lights (25.06\% THCA mean) (Figure 4). None of these physiological parameters were significantly different between any of the cultivars used in this study; therefore, data was pooled (data not shown).

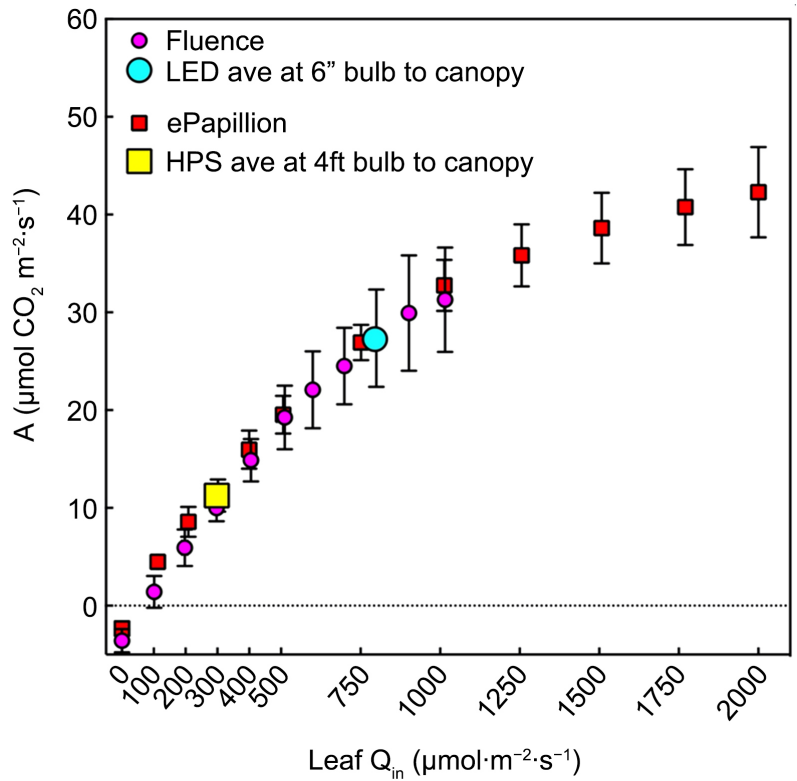

Figure 3. Light response curves measured at 0, 100, 200, 300, 400, 500, 750, 1000, 1250, 1500, 1750 and $2000 \mu \mathrm{mol} \cdot \mathrm{m}^{-2} \cdot \mathrm{s}^{-1}$ for $C$. sativa using HPS (ePapillion $1000 \mathrm{w}$ Double Ended bulbs) and LED (Fluence Bioengineering (now Fluence by Osram) SPYDR xPLUS) as the light sources (response curves for LED could not be measured above 1000 PPFD due to inability to fit the LiCor chamber between canopy and bulb).

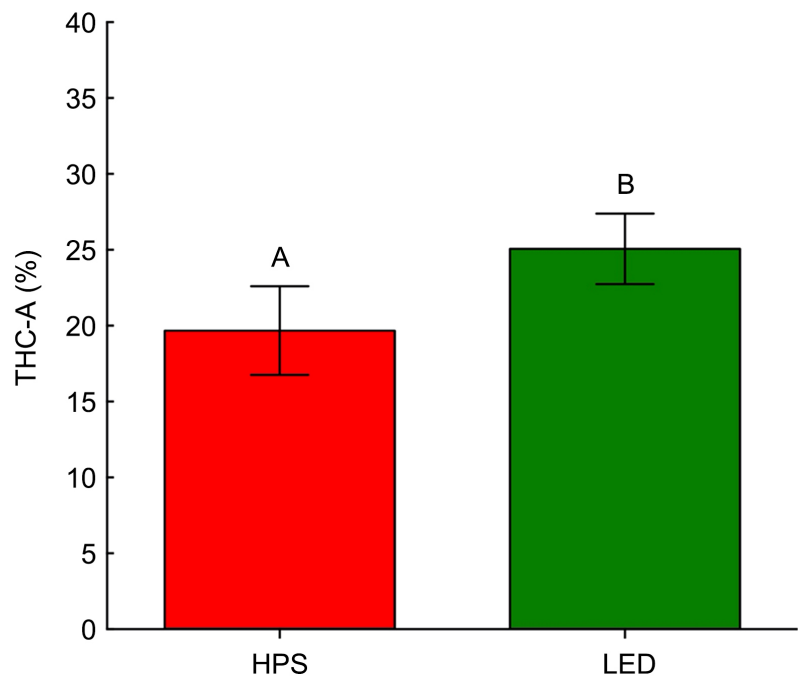

Figure 4. Potency means of 11 cultivars grown with HPS (ePapillion 1000w Double Ended bulbs) and LED (Fluence Bioengineering (now Fluence by Osram) (SPYDR xPLUS). Means with different letters are significantly different ( $T$-test $P<0.0001)$. 


\section{Discussion}

Lighting systems for cannabis cultivation operations serve two main purposes: provide the quality and quantity of light to achieve high levels of secondary metabolite production and maximizing plant biomass productivity. Lighting is often the most expensive component of controlled environmental cannabis cultivation facilities in terms of initial capital investment as well as ongoing expenses e.g., electricity, maintenance, bulb and lamp replacement [7]. The choice and dynamics of lighting system options, with consideration of physiological and photosynthetic enhancement, to provide enough light to enable maximum, uniform, and consistent productivity, while avoiding deleterious effects of high leaf temperatures [21] and photo-oxidative damage, when the absorption of light energy exceeds the capacity of photosynthesis [12], is an important design consideration for cannabis producers to evaluate; in order to not induce substantially higher lighting-related input costs while at the same time not limiting productivity. With the aim of understanding the response of cannabis productivity and cannabinoid potency, this study presents the results of extensive cannabis leaf photosynthetic measurements and cannabinoid potency results for a range of cultivars grown using LED and HPS illumination.

The first part of this study was to evaluate leading LED light manufacturers/models and identify which option provides the most uniform distribution of light; resulting $A$ and leaf temperature. Uniformity of light at the leaf and canopy level is an important determinant to achieve a consistent sellable product to meet market demand and customer expectations in the regulated cannabis industry. Figure 1 shows LED lighting options with a range of light uniformity across the canopy space these lights claim to cover. Fluence by Osram SPYRD xPLUS model clearly demonstrated optimal light distribution and photosynthetic response. Although cannabis plants exhibit a high degree of plasticity with respect to intensity [19] [22] [23], spectrum of light [13] [17], and temperature, there is a general consensus that photosynthetic response for cannabis is limited above $30^{\circ} \mathrm{C}$ [20] [24]. The Fluence LED light also maintained leaf temperature within an optimal range to achieve maximum photosynthetic rates (Figure 2).

For the second part of this study, it was hypothesized that $C$. sativa grown under an LED light source that provides more optimal photon flux density and reduced thermal heat load than $C$. sativa grown under HPS illumination, will photosynthetically outperform and should, theoretically, have increased cannabinoid potency compared to $C$. sativa grown under traditional HPS lights. Fluence by Osram SPYDR XPLUS was chosen, based on the results from the first part of this study, to test physiological performance versus an HPS light. The photosynthetic results of this trail demonstrated cannabis' remarkable plasticity to absorb high incident light levels from HPS or LED light sources and successfully use this light energy to assimilate large amounts of carbon (Figure 3). These results are generally consistent with trends of other studies reporting in- 
creased cannabis photosynthetic response to elevated PPFD [6] [7] [20] [23]. However, a key difference between HPS and LED lights is the enhanced long-wave (heat) radiation HPS lights emit. The greater efficiency of LED lights enables high PPFD cultivation with LED bulbs mounted much closer (6 inches) to the canopy without the induction of deleterious leaf temperature impacts, as opposed to HPS lights which require $4 \mathrm{ft}$ of spacing between bulb and canopy, which yields much lower PPFD. In our study, Fluence by Osram SPYDR xPLUS LED light produced $167 \%$ increase of photosynthetically active radiation (795 PPFD under LED versus 298 under HPS), $142 \%$ increased photosynthetic rate (11.26 under LED versus $27.26 A \mu \mathrm{mol} \mathrm{CO}_{2} \mathrm{~m}^{-2} \cdot \mathrm{s}^{-1}$ under HPS) at the recommended distance compared to ePapillion $1000 \mathrm{w}$ Double Ended HPS (Figure 3).

Light response curves are generally designed to allow photosynthetic responses to increased irradiance, but not enough time between light intervals to allow leaf tissue to respond to changes in leaf temperature, as was the case in this study. The results shown in Figure 2, regarding leaf temperature and Figure 3, regarding distance from bulb to canopy and corresponding photosynthetic rates, highlight this point. In this study, the cultivars of cannabis did not photosynthetically respond different to LED or HPS lights or the intensity of light (Figure 3 ); however, if the leaf tissue was allowed to adapt to the temperature difference induced by these light sources photosynthetic rates would most likely become limited.

Phytochemistry in cannabis is very complex; more than 480 compounds have been identified [25], some belonging to primary metabolism, e.g., amino acids, fatty acids, and steroids; while others are secondary metabolites e.g., cannabinoids, terpenoids, flavonoids, stilbenoids, lignans, alkaloids, and hormones. The concentration of these compounds depends on tissue type, developmental stage (age), genetic or cultivar variety, and growth conditions (irradiance, nutrient uptake, atmospheric conditions, water conditions) as well as harvest and post-harvest processing. In this study, all cannabis cultivars grown under LED lights yielded an increase in THCA\% with an average increase of 5.39\% (mean of 25.06\% THCA under LED versus 19.67\% under HPS) (Figure 4). The importance of determining the conditions for optimal photosynthetic performance, maximum potency, designing, and operating a cultivation system that consistently maintains these conditions cannot be understated in the cannabis industry-since the majority of product is sold based on weight and cannabinoid potency.

Cultivation of cannabis in controlled environmental facilities is an energy intensive and capital expensive endeavor. To be successful in the cannabis industry operators must accomplish a combination of difficult tasks-not only follow and obey the strictest sets of rules and regulations for any cultivated plant (which are constantly changing and vary depending on location), but also meet market demands for quality and price (which again are constantly changing). In addition, these tasks must all too often be achieved with limited scientific knowledge re- 
garding cannabis cultivation. A key component of a healthy and successful controlled environmental cannabis cultivation facility is an operation that employs testing, data collection, analyses, and environmental research toward the discovery of the ideal conditions to set the cultivation environment. Additionally, this means understanding that specific cultivars may respond differently to minor modification to their environment. The ultimate goal is to achieve optimal conditions to achieve maximum genetic potential for all chosen cultivars and to meet changing market demands. The results of this study can be used to aid cannabis producers and the cannabis industry regarding factors to consider when making decision on lighting systems and further scientific investigation regarding cannabis' response to lighting in commercial scale cannabis cultivation facilities.

\section{Conflicts of Interest}

The authors declare no conflicts of interest regarding the publication of this paper.

\section{References}

[1] Lawlor, D.W. (1995) Photosynthesis, Productivity and Environment. Journal of EXperimental Botany, 46, 1449-1461. https://doi.org/10.1093/jxb/46.special_issue.1449

[2] Casal, J.J. and Yanovsky, M.J. (2005) Regulation of Gene Expression by Light. International Journal of Developmental Biology, 49, 501-511. https://doi.org/10.1387/ijdb.051973jc

[3] Casal, J.J., Fankhauser, C., Coupland, G. and Blázquez, M.A. (2004) Signalling for Developmental Plasticity. Trends in Plant Science, 9, No. 6.

https://doi.org/10.1016/j.tplants.2004.04.007

[4] Chen, M., et al. (2010) Arabidopsis HEMERA/pTAC12 Initiates Photomorphogenesis by Phytochromes. Cell, 141, 1230-1240. https://doi.org/10.1016/j.cell.2010.05.007

[5] (2019) The Global Cannabis Report.

[6] Lydon, J., Teramura, A.H. and Coffman, C.B. (1987) UV-B Radiation Effects on Photosynthesis, Growth and Cannabinoid Production of Two Cannabis sativa Chemotypes. Photochemistry and Photobiology, 46, 201-206. https://doi.org/10.1111/j.1751-1097.1987.tb04757.x

[7] Tang, K., Struik, P.C., Amaducci, S., Stomph, T.-J. and Yin, X. (2017) Hemp (Cannabis sativa L.) Leaf Photosynthesis in Relation to Nitrogen Content and Temperature: Implications for Hemp as a Bio-Economically Sustainable Crop. GCB Bioenergy, 9, 1573-1587. https://doi.org/10.1111/gcbb.12451

[8] Bourget, C.M. (2008) An Introduction to Light-Emitting Diodes. Hort Science, 43, 1944-1946.

[9] Massa, G.D., Kim, H.-H., Wheeler, R.M. and Mitchell, C.A. (2008) Plant Productivity in Response to LED Lighting. Hort Science, 43, 1951-1956.

[10] Morrow, R.C. (2008) LED Lighting in Horticulture.

[11] Hogewoning, S.W. (2012) On the Photosynthetic and Developmental Responses of Leaves to the Spectral Composition of Light. 
[12] Demmig-Adams, B. and Adams III, W.W. (1992) Photoprotection and Other Responses of Plants to High Light Stress. Annual Review of Plant Physiology and Plant Molecular Biology, 43, 599-626.

http://www.annualreviews.org/ https://doi.org/10.1146/annurev.pp.43.060192.003123

[13] Bilodeau, S.E., Wu, B.-S., Rufyikiri, A.-S., MacPherson, S. and Lefsrud, M. (2019) An Update on Plant Photobiology and Implications for Cannabis Production. Frontiers in Plant Science, 10. https://doi.org/10.3389/fpls.2019.00296

[14] Backer, R., Schwinghamer, T., Rosenbaum, P., et al. (2019) Closing the Yield Gap for Cannabis: A Meta-Analysis of Factors Determining Cannabis Yield. Frontiers in Plant Science, 10. https://doi.org/10.3389/fpls.2019.00495

[15] Hawley, D., Graham, T., Stasiak, M. and Dixon, M. (2018) Improving Cannabis Bud Quality and Yield with Subcanopy Lighting. HortScience, 53, 1593-1599.

https://doi.org/10.21273/HORTSCI13173-18

[16] Lalge, A., Cerny, P., Trojan, V. and Vyhnanek, T. (2017) The Effects of Red, Blue and White Light on the Growth and Development of Cannabis sativa L. Brno, Czech Republic.

[17] Magagnini, G., Grassi, G. and Kotiranta, S. (2018) The Effect of Light Spectrum on the Morphology and Cannabinoid Content of Cannabis sativa L. Medical Cannabis and Cannabinoids, 1, 19-27. https://doi.org/10.1159/000489030

[18] Moher, M., Jones, M. and Zheng, Y. (2021) Photoperiodic Response of in Vitro Cannabis sativa Plants. HortScience, 56, 108-113.

https://doi.org/10.21273/HORTSCI15452-20

[19] Potter, D.J. and Duncombe, P. (2012) The Effect of Electrical Lighting Power and Irradiance on Indoor-Grown Cannabis Potency and Yield. Journal of Forensic Sciences, 57, 618-622. https://doi.org/10.1111/j.1556-4029.2011.02024.x

[20] Chandra, S., Lata, H., Khan, I.A. and Elsohly, M.A. (2008) Photosynthetic Response of Cannabis sativa L. to Variations in Photosynthetic Photon Flux Densities, Temperature and $\mathrm{CO}_{2}$ Conditions. Physiology and Molecular Biology of Plants, 14, 299-306. https://doi.org/10.1007/s12298-008-0027-x

[21] Greer, D.H., Berry, J.A. and Björkman, O. (1986) Photoinhibition of Photosynthesis in Intact Bean Leaves: Role of Light and Temperature, and Requirement for Chloroplast-Protein Synthesis during Recovery. Planta, 168, 253-260.

[22] Vanhove, W., van Damme, P. and Meert, N. (2011) Factors Determining Yield and Quality of Illicit Indoor Cannabis (Cannabis spp.) Production. Forensic Science International, 212, 158-163. https://doi.org/10.1016/j.forsciint.2011.06.006

[23] Morrison, V.R., Llewellyn, D. and Zheng, Y. (2021) Cannabis Yield, Potency, and Leaf Photosynthesis Respond Differently to 1 Increasing Light Levels in an Indoor Environment .

[24] Bazzaz, F.A., Dusek, D., Seigler, D.S. and Haney, A.W. (1975) Photosynthesis and Cannabinoid Content of Temperate and Tropical Populations of Cannabis sativa. Biochemical Systematics and Ecology, 3, 15-18. https://doi.org/10.1016/0305-1978(75)90036-8

[25] Wu, B.-S., Hitti, Y., MacPherson, S., Orsat, V. and Lefsrud, M.G. (2020) Comparison and Perspective of Conventional and LED Lighting for Photobiology and Industry Applications. Environmental and Experimental Botany, 171, 103953. https://doi.org/10.1016/j.envexpbot.2019.103953 